\title{
Oral Lecithin and Linoleic Acid in Friedreich's Ataxia: II. Clinical Results
}

\author{
S.B. MElanCon, M. vanasse, G. GeOfFroy, L. BARABE, A. ProulX, G. FONTAine, L. DALLAiRE, M. POTIER
}

SUMMARY: Twenty-two patients with Friedreich's Ataxia and ten normal controls were followed for one year and assessed as to their clinical performance after two successive six-month periods of lecithin or safflower oil. Results demonstrated no significant difference in performance scores according to group assignation, neither in patients nor in controls. According to stages, two patients in stage $I$ and to a lesser degree, one patient in stage IV showed better scores for muscle strength and some motor accuracy and coordination tests with lecithin. Controls as groups maintained positive scores in all tests. Patients as

RÉSUMÉ: Nous avons suivi l'évolution de vingt-deux ataxiques et de dix témoins normaux durant un an et mesuré leur performance clinique après deux périodes de six mois suivant la prise de lécithine ou d'huile de carthame. Les résultats ne démontrèrent aucune différence dans la moyenne de performance des groupes de patients comparés entre eux.

Selon les stades fonctionnels, deux patients de stade I et à un moindre degré, un patient de stade $I V$, améliorèrent leur force musculaire et leur coordination motrice avec la lécithine. Les témoins, comme groupes, ont amélioré leur performance dans toutes les épreuves auxquelles ils furent soumis. Chez les patients, par groupes, seuls les résultats des tests de dynamométrie et de coordination s'avé- groups showed negative mean values in nine out of eleven tests. Again as groups, patients receiving safflower oil demonstrated a mean $8 \%$ less deterioration than patients receiving lecithin. This study demonstrates that objective clinical tests and the participation of normal controls are a must in a therapeutic trial implicating patients with a progressive disorder such as Friedreich's Ataxia. The possible role of linoleic acid as the active factor from which clinical improvement proceeded in some specific patients and with early functional stages of the disease, has to be considered and reevaluated in the near future.

rèrent améliorés. Les neuf autres paramètres cliniques se sont détériorés chez eux après supplémentation en lécithine ou en huile de carthame. Cette détérioration fut d'environ $8 \%$ moins importante après la période de prise d'huile de carthame. Cette étude met l'emphase sur la nécessité d'inclure des témoins normaux et des méthodes objectives d'évaluation clinique dans la réalisation d'un essai thérapeutique pour une maladie évolutive comme l'ataxie de Friedreich. Les résultats surprenant obtenus à l'aide de l'huile de carthame, remettent en question l'implication relative de la choline et de l'acide linoléique dans l'amélioration percue chez certains patients. Ceci devrait faire l'objet d'une réévaluation dans un avenir prochain.
Le centre de recherche pédiatrique, Hôpital SainteJustine, Université de Montreal.

Reprint requests for the entire supplement on Friedreich's Ataxia (Phase Three, Part Two) to: Prof. André Barbeau, Clinical Research Institute of Montreal, 110 West Pine Avenue, Montreal, Quebec, Canada, H2W IR?.

\section{INTRODUCTION}

In a recent publication, Livingstone et al, (1981) reported some functional improvement in upper limb coordination in three of seven patients with Friedreich's Ataxia, four of six patients with primary cerebellar degeneration and three of seven patients with mixed ataxia, after twelve weeks of oral choline chloride supplements. More recently, Pentland et al, (1981) failed to demonstrate any significant difference in upper limb functions (timed handwriting, spiral drawing and rapid repetitive finger movements) in twelve patients with Friedreich's Ataxia supplemented with pure lecithin during the first and the third month of a threemonth period.

The present study was initiated in the early months of 1980 and terminated in the summer of 1981 . Twenty-two patients with Friedreich's Ataxia and ten normal controls were given oral supplements of either lecithin or placebo in a double-blind crossover study which aimed at confirming previously published reports, stating some clinical improvements in muscle strength and coordination after lecithin therapy.

A detailed description of the patients, materials and methods will be found in the previous paper of this issue. We wish to report here the results of a thorough clinical appraisal and discuss the effect of lecithin on some specific clinical features of Friedreich's Ataxia.

\section{RESULTS}

The data were gathered according to group assignation (A or B) and functional stages ( 0 to $\mathrm{V})$. The abbreviations used in the text and table are as follows: $\mathrm{A}$ and $\mathrm{B}$ refer to patients 
group; $\mathrm{CA}$ and $\mathrm{cB}$ to controls for respective group; 1 or $r$ to left or right; $\mathrm{L}$ to lecithin and $\mathrm{P}$ to placebo (which was made of a linoleic acid-rich preparation of safflower oil). I refers to improved, $\mathrm{W}$ to worsened and $\mathrm{S}$ to stable.

\section{a) Joint mobility and musculoskeletal deformities}

We observed very little change in the degree of amplitude of the four joints studied (tables Ia and Ib). Flexion of the shoulder and flexion of the first toe were reduced during the second sixmonth period in both groups whether on lecithin or safflower oil. No noticeable deformities were observed in any patient during the twelve-month therapeutic trial.

\section{b) Muscle function}

Results of the semi-quantitative evaluation of muscle strength are shown in tables IIa and IIb. Safflower oil was more effective than lecithin in stabilizing the muscular performance in both groups: A and B and in stage I and II patients.

Results of the dynamometer studies are detailed in tables III to XI. Most patients improved their performance and did so to a greater extent after the second six-month period, whether on lecithin or safflower oil. Stage I patients demonstrated a significant mean improvement of $17 \%$ in muscle strength with lecithin in comparison with safflower oil (table XII). Stage II patients showed no significant difference in improvement with either supplement. Stage III patients had an apparent $9 \%$ mean improvement in muscle strength with lecithin but this result could be ascribed to two specific muscle functions: extension of the second finger (table VII) and eversion of the foot (table XI). With regards to this last function, their placebo score was the only negative mean value encountered during the dynamometer study. Our single stage IV patient showed a mixed pattern of improvement in favour of placebo for the upper extremities and lecithin for the lower extremities.

\section{c) Ataxia, coordination and motor accuracy}

The corridor test (table XIII) disclosed a marked difference between patients and controls; there was a slight advantage in favour of safflower oil over lecithin in both groups of patients, and to some extent in stage I patients. Comparable results were observed with repetitive movements of the foot (table XIV). The other two coordination and motor accuracy tests for the knee (table XV) and hip (XVI) showed deterioration in patient's performance after the second six-month period without regard for the supplement used. The timed stability tests were not altered by either lecithin or safflower oil supplements (table XVII). It was felt that stationary equilibrium was best evaluated with eyes closed in stage I patients, and with eyes opened in stages II and III patients.

\section{UPPER EXTREMITIES}

Results of the SCSIT motor accuracy test (table XVIII) revealed major improvement in accuracy scores after the second trial period. However when these scores were adjusted for time needed to complete the test, it became obvious that practice had little to do with the results. Safflower oil was more effective than lecithin in group A patients but less so in group B patients. According to stages, lecithin proved effective on accuracy alone. It was impossible to suggest any trend towards improvement by one or the other supplement with adjusted scores. The number of repetitive movements of the finger increased with time rather than with therapy except for the two stage I and the one stage IV patients on placebo (table XIX). Distal coordination improved (table XX) and proximal coordination deteriorated (table XXI) when group A patients went from lecithin to safflower oil. Group B patients were unaffected by the crossover. Patients from stages II and III appeared to benefit from safflower oil supplements with regards to distal coordination only (table XX). Results of the meter-nose test went to the advantage of lecithin for the normal controls and for the single stage IV patient (table XXII).

\section{d) Functional tests}

The climbing and sitting tests had to be eliminated from the original battery because of difficulty in interpretation and excessive fatigue and stress for some patients.

Results of five timed functional tests which could be correlated with the results of the questionnaire are given in tables XXIII and XXIV. The minus (-) sign means improvement over the pretrial evaluation. Running without or with support deteriorated slightly with repetition. On the contrary, the " $t$ shirt" and the "lacing" tests improved with practice. "Speech" was slightly improved in both groups of patients while on safflower oil. According to stages (table XXIV), lecithin was more effective with regards to "clothing" for stages I, II and III, while safflower oil was more valuable for "shoe lacing" and "speech" in stages II, III and IV.

The untimed functional tests results are given in table XXV. There was an overall loss in mean achievement for both groups. Lecithin was more effective than safflower oil in minimizing the ill-effects of the disease according to group assignation. There was however no trend towards improvement with lecithin according to stages other than stage IV.

\section{e) Questionnaire}

In answer to the questionnaire, patients in group A felt that they were improved with either supplements, while patients in group B felt deteriorated (table XXVI). According to stages, safflower oil was subjectively more effective than lecithin. Details of the answers appear in table XXVII. Difficulties in transfer and hygiene were of major concern to patients and parents. Answers as to feelings of improvement or worsening for feeding, clothing and speech were in agreement with data gathered through quantitative tests (table XXIII). The subjective improvement in handwriting was not in agreement with the results of the SCSIT test (table XVIII) which showed an equal number of gain and loss.

A summary of the mean percent changes in all clinical parameters tested over a one-year period is shown in table XXVIII. This summary demon- 
TABLE Ia

PERCENT CHANGES IN JOINT MOBIITY BY GROUP OF PATIENTS

AND SINGIE ARTICUIATION

\begin{tabular}{|c|c|c|c|c|c|c|}
\hline \multirow[t]{2}{*}{ SHOULDER } & \multicolumn{2}{|c|}{ Flexion } & \multicolumn{2}{|c|}{ Int. Rotation } & \multicolumn{2}{|c|}{ Ext. Rotation } \\
\hline & left & right & left & right & left & right \\
\hline AL & -2.7 & -0.3 & 0 & 0 & -2.7 & 0 \\
\hline AP. & -6.8 & -1.1 & 0 & 0 & 0 & 0 \\
\hline BP & -7.7 & -1.8 & -0.9 & -1.8 & 0 & 0 \\
\hline BLL & -11.5 & -9.1 & -0.9 & -0.9 & 0 & 0 \\
\hline
\end{tabular}

\begin{tabular}{ccccccc} 
HIP & \multicolumn{2}{c}{ Extension } & \multicolumn{2}{c}{ Int. Potation } & \multicolumn{2}{c}{ Ext. Rotation } \\
AL & left & right & left & right & left & right \\
AP & 0 & 0 & 0 & +1.4 & 0 & 0 \\
BP & +4.5 & +5.4 & 0 & 0 & 0 & 0 \\
BL & -1.4 & -1.4 & 0 & 0 & +1.4 & +0.9 \\
& -0.9 & +0.9 & -1.4 & -1.4 & +0.9 & 0
\end{tabular}

\begin{tabular}{lccccccc}
\hline \multirow{2}{*}{ ANKLE } & Dorsal flexion & \multicolumn{2}{c}{ Eversion } & & \multicolumn{2}{c}{ Inversion } \\
\cline { 2 - 8 } & left & right & left & right & left & right \\
AP & +0.9 & -1.1 & 0 & +0.9 & -2.3 & -2.7 \\
BP & +0.2 & -0.9 & 0 & 0 & +0.9 & -0.9 \\
BL & +2.7 & +4.1 & 0 & 0 & 0 & 0
\end{tabular}

\begin{tabular}{lll}
\hline FIRST TOE & \multicolumn{2}{c}{ Flexion } \\
& \multicolumn{1}{l}{ Left } & Right \\
AL & +1.1 & -1.4 \\
AP & -2.4 & -3.6 \\
BP & -3.4 & +0.4 \\
BL & -7.3 & +2.3
\end{tabular}

TABLE $\mathrm{nb}$

MEAN PETCENT CHANGE IN JODNT MOBIITY (ALL ARTICULATIONS AND AIL PATIENTS GROUPED)

$\begin{array}{lcc}\text { JoINT } & \underline{P \text { (Safflower) }} & \text { L (Lecithin) } \\ \text { Shoulder } & -1.68 & -2.38 \\ \text { Hip } & +0.78 & 0.08 \\ \text { Ankle } & +0.58 & -0.98 \\ \text { First toe } & -2.28 & -1.38\end{array}$

TARUE IIO

PERCENT CHANGE IN MUSCLE STREATTH (SIMI-QUNNTITATTVE) ACOORUING TO GROUP ASSIGATION

\begin{tabular}{lllll} 
GROUP & MEAN & \multicolumn{1}{c}{$\frac{\text { OUTCOME }}{W}$} & \\
AL & -2.28 & $(-20$ to +5.4$)$ & $I=5 \quad W=6$ \\
AP & -1.78 & $(-9.6$ to +8.6$)$ & $I=5 \quad W=6$ \\
BP & +1.58 & $(-6.2$ to +9.4$)$ & $I=5 \quad W=5 \quad S=1$ \\
EL & -4.18 & $(-9.7$ to 3.0$)$ & $I=0 \quad W=9 \quad \mathrm{~S}=2$ \\
\hline
\end{tabular}

TABLE ITb

MEAN PERCFNT CHNMEE IN MUSCTE STFENCTH (SEMI-CUANTITATTVE) ACCOROING TO STAGES

\begin{tabular}{|c|c|c|c|}
\hline STAGES & $\underline{n}$ & $\underline{P}$ (SAFFLONER) & $L$ (LECITHIN) \\
\hline I & (2) & +6.18 & -2.08 \\
\hline II & (11) & +1.48 & -1.88 \\
\hline III & (8) & -3.88 & -6.38 \\
\hline N V & (1) & -4.58 & -3.78 \\
\hline
\end{tabular}

TABLE III

QUANTITATIVE MUSCLE STPENKTH EVALUATION IN FRILDREICH'S ATMXIA: FLXION OF THE NECK

\begin{tabular}{|c|c|c|c|}
\hline GRRUP & MEAN & RANGE & $\underline{\alpha T C O M E}$ \\
\hline AL & +29.48 & $(-18$ to +1088$)$ & $I=9 \quad W=2$ \\
\hline AP & +46.98 & $(-13$ to +1588$)$ & $I=8 \quad W=3$ \\
\hline $\mathrm{BP}$ & +26.78 & $(-31$ to +1668$)$ & $I-8 \quad W=3$ \\
\hline BL & +62.08 & $(-14$ to +3008$)$ & $I=9 \quad W=2$ \\
\hline SINGES & $\underline{n}$ & P (Safflower) & $\underline{L}$ (lecithin) \\
\hline I & 2 & +268 & +228 \\
\hline II & 11 & +358 & +578 \\
\hline II I & 8 & +278 & +248 \\
\hline IV & 1 & +1588 & +1088 \\
\hline
\end{tabular}

TABLE IV

QANTITATIVE MUSCIE STRENGTH EVALUATION IN FRIFDREICH'S ATAXIA: ABDUCTION OF THE SHOULDERS

\begin{tabular}{|c|c|c|c|c|c|}
\hline GROUP & MEAN & RANGE & & TOOME & \\
\hline ALLl & +52.78 & $(-12$ to $+182 t)$ & $I=10$ & $w=1$ & \\
\hline Alr & +64.68 & $(-0$ to +1908$)$ & $I=9$ & $W=0$ & $S=2$ \\
\hline & +57.18 & & & & \\
\hline API & +54.48 & $(-7$ to +1368$)$ & $I=9$ & $w=2$ & \\
\hline $\mathrm{APr}$ & +60.58 & $(-4$ to +1268$)$ & $I=9$ & $w=1$ & $s=1$ \\
\hline & +57.48 & & & & \\
\hline $\mathrm{BPI}$ & +45.58 & $(-20$ to +1898$)$ & $I=9$ & $W=2$ & \\
\hline $\mathrm{BPr}$ & +86.78 & $(-19$ to +3338$)$ & $I=9$ & $w=1$ & $s=1$ \\
\hline & +66.18 & & & & \\
\hline
\end{tabular}

\begin{tabular}{|c|c|c|c|}
\hline BLil & +67.38 & $(-10$ to $+187 z)$ & $I=9 \quad W=2$ \\
\hline \multirow[t]{2}{*}{$\mathrm{BL}, \mathrm{r}$} & +94.48 & $(-0$ to +3508$)$ & $I=11$ \\
\hline & +80.88 & & \\
\hline
\end{tabular}

\begin{tabular}{lccc} 
STAGFS & n & P (Safflower) & $\frac{\text { L (lecithin) }}{+148}$ \\
I & 2 & +698 & +368 \\
II & 11 & +468 & +798 \\
III & 8 & +1588 & +478 \\
IV & 1 & +1868 \\
\hline
\end{tabular}


TABLE V

QUANTITATIVE MUSCTE STRENGT: EVAIUATIOUN IN FRIEDREICH'S ATNYIA: ABDUCTION OF THE FIFTH FTNGER

\begin{tabular}{|c|c|c|c|}
\hline Group & MEAN & RANGE & QUTCONE \\
\hline A.t. & +528 & $(-13$ to +1608$)$ & $\mathrm{I}=8 \mathrm{~W}=3$ \\
\hline \multirow[t]{2}{*}{ ALr. } & +218 & $(-45$ to +3008$)$ & $I-5 \mathrm{~W}=5 \mathrm{~S}=1$ \\
\hline & +368 & & \\
\hline AP1 & +998 & $(+6$ to +2708$)$ & $I=11$ \\
\hline \multirow[t]{2}{*}{ APr } & +628 & ( 0 to +2608$)$ & $I=10$ \\
\hline & +808 & & \\
\hline BP1 & +368 & $(-14$ to +1208$)$ & $I=10 \mathrm{~W}=1$ \\
\hline \multirow[t]{2}{*}{$\mathrm{BPY}$} & +438 & $(-45$ to +1568$)$ & $I=8 \mathrm{~W}=1 \mathrm{~S}=2$ \\
\hline & +398 & & \\
\hline BLI & +788 & $(-9$ to +1668$)$ & $I=9 \mathrm{~W}=1 \mathrm{~S}=1$ \\
\hline \multirow[t]{2}{*}{ BLIr } & +838 & (- 7 to +3208$)$ & $I-10 \mathrm{~W}=1$ \\
\hline & +808 & & \\
\hline
\end{tabular}

\begin{tabular}{lccc}
\hline STAGES & n & $\frac{\text { g(Safflower) }}{\text { L (Lecithin) }}$ \\
I & 2 & +78 & +398 \\
II & 11 & +688 & +558 \\
III & 8 & +468 & +658 \\
IV & 1 & +1218 & +558
\end{tabular}

TABLE VII

QUAMTTAATIVE MUSCLE STRENGT! EVALUATION IN FRIEDRETCT'SATAXIA AND NORMAL CONTROLS: EXTENSION OF THE SBCOND FDNGER

\begin{tabular}{|c|c|c|c|c|}
\hline GROUP & MEAN & RANCE & aur & COME \\
\hline AL & +448 & $(-23$ to +2338$)$ & $I=9$ & $w=2$ \\
\hline CAL & +268 & $(+21$ to +368$)$ & $I=4$ & \\
\hline AP & +728 & $(-20$ to +4718$)$ & $I=8$ & $w=1$ \\
\hline CAP & +568 & $(0$ to +1078$)$ & $I=3$ & \\
\hline $\mathrm{BP}$ & +558 & $(-27$ to +2008$)$ & $I=10$ & $w=1$ \\
\hline CBP & +208 & $(+8$ to +268$)$ & $I=6$ & \\
\hline BL & +1178 & $(-38$ to +2338$)$ & $I=10$ & $w=1$ \\
\hline CBL & +558 & $(+20$ to +908$)$ & $I=4$ & \\
\hline
\end{tabular}

\section{TABLE VI}

QUANTITATIVE MUSCIE STRZNGT' EVILUATION DN FRIEDREICH'S ATAXIA:

\section{PAIMAR IITEROSSBOUS (SECOND FIMGER)}

\begin{tabular}{|c|c|c|c|}
\hline GROUP & MEAN & RANGE & OUTCOME \\
\hline ALI & +218 & $(-54$ to +858$)$ & $I=7 \quad W=4$ \\
\hline \multirow[t]{2}{*}{ ALr } & +218 & $(-45$ to +1008$)$ & $I=7 \quad W-4$ \\
\hline & +218 & & \\
\hline AP1 & +508 & (- 8 to +1408$)$ & $I=10 \quad \mathrm{~b}=1$ \\
\hline \multirow[t]{2}{*}{ APr } & +638 & $(-31$ to +2758$)$ & $I=8 \quad W=3$ \\
\hline & +568 & & \\
\hline $\mathrm{BP} 1$ & +538 & $(-33$ to +2008$)$ & $I=9 \mathrm{~W}=2$ \\
\hline \multirow[t]{2}{*}{$\mathrm{BPr}$} & +638 & $(-13$ to +1608$)$ & $I=9 \quad \mathrm{~W}=1 \quad \mathrm{~S}=1$ \\
\hline & +588 & & \\
\hline BLI & +668 & $(-40$ to +2663$)$ & $I=9 \quad W=2$ \\
\hline \multirow[t]{2}{*}{ BLr } & +878 & $(+2$ to +2608$)$ & $I=11$ \\
\hline & +760 & & \\
\hline STAGES & $\underline{n}$ & P (Saffiaier) & $\underline{L}$ (Lecithin) \\
\hline I & 2 & +268 & +399 \\
\hline II & 11 & +658 & +498 \\
\hline III & 8 & +503 & +408 \\
\hline IV & 1 & +658 & +628 \\
\hline
\end{tabular}

$\begin{array}{lrcc}\text { STAGES } & \text { n } & \frac{\text { P (Safflower) }}{\text { L (Lecithin) }} \\ 0 & 10 & +348 & +338 \\ \text { I } & 2 & +368 & +638 \\ \text { II } & 11 & +748 & +708 \\ \text { III } & 8 & +328 & +778 \\ \text { IV } & 1 & +2698 & +1098\end{array}$

TABIE VIII

QUANTTTATIVE MUSCLE SIRENGTH EVALATION IN FRIDDRETCF' SATEXIA: ABDUCTION OF TRE HIP

\begin{tabular}{|c|c|c|c|c|c|}
\hline GROPP & MEAN & & NGE & OUTOOME & \\
\hline ÁLl & +1408 & $(-5$ & $t 0+800)$ & $I=8 W=2$ & $s=1$ \\
\hline ALr & +1358 & 10 & to +780$)$ & $I=10 \quad W=1$ & \\
\hline & +1378 & & & & \\
\hline AP1 & +478 & $(-10$ & to +85$)$ & $I=8 \quad W=1$ & \\
\hline APr & +678 & $(-46$ & to $+200 !$ & $I=9 W=1$ & \\
\hline & +578 & & & & \\
\hline $\mathrm{BP1}$ & +638 & $(-2)$ & to + 440) & $I=8 \quad W=2$ & $s=1$ \\
\hline $\mathrm{BPr}$ & +968 & $(-15$ & to +440) & $I=7 \mathrm{~W}=3$ & $s=1$ \\
\hline & +798 & & & & \\
\hline BLI & +808 & 10 & to + 240) & $I=9$ & \\
\hline BLIT & +718 & $(-42)$ & to +300$)$ & $I=\theta \quad W=1$ & \\
\hline & +758 & & & & \\
\hline STAGES & $\underline{n}$ & $\underline{P}$ (S & afflower) & $L$ (Lecithin) & \\
\hline I & 2 & & 308 & +388 & \\
\hline II & 11 & & 618 & +668 & \\
\hline III & 6 & & 878 & +638 & \\
\hline IV & 1 & & 1108 & +7808 & \\
\hline
\end{tabular}


TABLE IX

QUANCITATTVE MUSCTE STRENGTY EVRLUATION IN FRIEDREICH SATAXIA: EXTERSION OF TTE KNEE

\begin{tabular}{|c|c|c|c|c|c|}
\hline \multirow{2}{*}{$\frac{\text { GrouP }}{\text { ALLl }}$} & \multirow{2}{*}{$\frac{\text { MEAN }}{+188}$} & \multicolumn{2}{|c|}{ RANGE } & \multicolumn{2}{|c|}{ QTTCOME } \\
\hline & & $(-38$ & to +77$)$ & $I=8$ & $\mathrm{~W}=3$ \\
\hline \multirow[t]{2}{*}{ ALr. } & +248 & $(-25$ & to +81$)$ & $I=9$ & $W=2$ \\
\hline & +218 & & & & \\
\hline API & +318 & $(-45$ & to +96$)$ & $I=8$ & $w=2$ \\
\hline \multirow[t]{2}{*}{$\mathrm{APr}$} & +338 & $(-50$ & to +84 ) & $I=9$ & $W=1$ \\
\hline & +328 & & & & \\
\hline BPI & +748 & $1+5$ & to +322$)$ & $I=11$ & \\
\hline \multirow[t]{2}{*}{ BPr } & +27 & $\{-26$ & to +94$)$ & $I=10$ & \\
\hline & +508 & & & & \\
\hline $\mathrm{BLL}$ & +1088 & 10 & to + 374) & $I=10$ & $w=1$ \\
\hline \multirow[t]{2}{*}{ BLr } & +458 & $1-36$ & to +133$)$ & $I=8$ & $w=3$ \\
\hline & +768 & & & & \\
\hline STAGES & $\underline{n}$ & $\underline{P}$ & (Safflower) & $\underline{L}$ (Le & aithin) \\
\hline I & 2 & & +78 & & 298 \\
\hline II & 10 & & +458 & + & 458 \\
\hline III & 8 & & +498 & + & 558 \\
\hline IV & 1 & & +188 & + & 288 \\
\hline
\end{tabular}

TABLE XI

QUANTTTATIVE MUSCLE STRENGTI EVALUATION IN FRIEDREICH'S ATAXIA AND NORMAL CONIPOLS: EVERSION OF TIE FOOT (PERONEAL)

$\begin{array}{llll}\text { GROUP } & \text { MEAN } & \text { RANGE } & \text { OJTCOME } \\ \text { AL } & +428 & (-53 \text { to }+1368) & I=7 \quad W=2 \\ \text { CAL } & +598 & (+66 \text { to }+1128) & I=2 \\ \text { AP } & +388 & (-79 \text { to }+1508) & I=7 \quad W=2 \\ \mathrm{CAP} & +1158 & (+92 \text { to }+1538) & I=2 \\ \mathrm{BP} & +108 & (-100 \text { to }+1508) & I=6 \quad \mathrm{~W}=4 \\ \mathrm{CBP} & +428 & (+12 \text { to }+788) & I=3 \\ \mathrm{BL} & +638 & (-2 \text { to }+1338) & \mathrm{I}=9 \mathrm{~W}=2 \\ \mathrm{CBL} & +628 & (+41 \text { to }+888) & I=2\end{array}$

\begin{tabular}{|c|c|c|c|}
\hline STAGES & $\underline{n}$ & P (Safflower) & L (Lecithin) \\
\hline 0 & 5 & +718 & +608 \\
\hline $\mathbf{I}$ & 2 & +58 & +318 \\
\hline II & 11 & +448 & +638 \\
\hline III & 6 & -128 & +388 \\
\hline IV & 1 & NP* & $\mathrm{NP}^{*}$ \\
\hline
\end{tabular}

* Not possible

\section{TABLE X}

QUANTITATTVE MUSCLE STRENGM EVALIATION IN FRIEDREICH'S ATAXIA ARD NORMAL CONTROIS: DORSIFLEXION OF THE FOOT

\begin{tabular}{|c|c|c|c|c|c|}
\hline GRoup & MEAN & RANGE & \multicolumn{3}{|c|}{ QATCOME } \\
\hline AL & +11.78 & $\{-36$ to +808$\}$ & $I=6$ & $w=2$ & $s=1$ \\
\hline CAL & +21.28 & $(-10$ to +738$)$ & $I=3$ & $W=1$ & \\
\hline AP & +12.28 & $(-31$ to +1058$)$ & $I=4$ & $w=4$ & $s=1$ \\
\hline CAP & +58.88 & $(+1$ to +1708$)$ & $I=3$ & & \\
\hline$B P$ & +28.18 & $(-100$ to +1008$)$ & $I=9$ & & $s=1$ \\
\hline CBP & +28.08 & $(-27$ to +628$)$ & $I=4$ & $w=1$ & \\
\hline BL & +24.28 & $(-54$ to +748$)$ & $I=9$ & $w=1$ & \\
\hline $\mathrm{CBL}$ & +20.08 & $\{-35$ to +628$\}$ & $I=3$ & $w=1$ & \\
\hline
\end{tabular}

\begin{tabular}{|c|c|c|c|}
\hline STAGES & $\underline{n}$ & P (Safflower) & I (Lecithin) \\
\hline 0 & 9 & +398 & +208 \\
\hline I & 2 & +108 & +228 \\
\hline II & 11 & +248 & +228 \\
\hline III & 5 & +128 & +48 \\
\hline IV & 1 & $\mathrm{NP}^{*}$ & $N^{*}$ \\
\hline
\end{tabular}

* Not possible
TABLE XII

QUAMTITTIVE MUSCIE STREXGTH EVALATION IN FRIEDREICH'S ATAXIA: CUNULATIVE MEN PERCENT IMPRONEMENT IN NINE MUSCLE RLNCTIONS VTTH SAFFIOWER OIL (P) AND LECITHEN (L) ACCORDING TO FUNCTIOMAL STAGE

\begin{tabular}{|c|c|c|c|c|c|c|c|c|c|c|c|c|}
\hline \multirow[b]{2}{*}{ NUSCLE FUNCTION } & \multicolumn{2}{|c|}{0} & \multicolumn{2}{|c|}{ I } & \multicolumn{2}{|c|}{ II } & \multicolumn{2}{|c|}{ III } & \multicolumn{2}{|c|}{ IV } & \multicolumn{2}{|c|}{$I-I V$} \\
\hline & $P$ & $\mathbf{L}$ & $D$ & $I_{1}$ & $P$ & $\mathrm{~L}$ & $\mathbf{P}$ & L & $\mathbf{P}$ & L & $\mathbf{P}$ & L \\
\hline Neck Flexion & - & - & 26 & 22 & 35 & 57 & 27 & 24 & 158 & 108 & 36 & 44 \\
\hline Shoulder Abruction & - & - & 14 & 36 & 69 & 79 & 46 & 47 & 158 & 186 & 60 & 68 \\
\hline Finger Abduction & - & - & 7 & 39 & 68 & 55 & 46 & 65 & 121 & 55 & 57 & 57 \\
\hline Palmar Interosseous & - & - & 36 & 39 & 65 & 49 & 50 & 40 & 65 & 62 & 56 & 45 \\
\hline Finger Extension & 34 & 33 & 26 & 63 & 74 & 70 & 32 & 77 & 269 & 109 & 64 & 74 \\
\hline Hip Abduction & - & - & 30 & 38 & 61 & 66 & 37 & 63 & 110 & 780 & 68 & 98 \\
\hline Knee Extension & - & - & 7 & 29 & 45 & 45 & 49 & 55 & 18 & 28 & 42 & 46 \\
\hline Foot Dorsifleyion & 39 & 20 & 10 & 22 & 24 & 22 & 12 & 4 & - & - & 19 & 27 \\
\hline Foot Eversion & 71 & 70 & 5 & 31 & 44 & 63 & -12 & 38 & - & - & 22 & 52 \\
\hline MEAN TOLAL GNIN & 48 & 38 & $18^{*}$ & 35" & 54 & 56 & 37 & 46 & 128 & 190 & 47 & 56 \\
\hline$I S D=S D$ & - & - & \pm 12 & \pm 12 & \pm 1.7 & \pm 17 & \pm 28 & \pm 22 & \pm 80 & \pm 265 & \pm 18 & \pm 23 \\
\hline
\end{tabular}


TARIE XIII

\section{MEAN CHANGES IN STEADINESS OF GAIT (CORRIDOR TEST)}

$\begin{array}{cc}\text { GROP } & \underline{\text { MEAN }} \\ \mathrm{AL} & -10.0 \mathrm{~cm} \\ \mathrm{CAL} & +2.5 \mathrm{~cm} \\ \mathrm{AP} & -5.0 \mathrm{~cm} \\ \mathrm{CAP} & +3.3 \mathrm{~cm} \\ \mathrm{BP} & -53.7 \mathrm{~cm} \\ \mathrm{CBP} & +2.0 \mathrm{~cm} \\ \mathrm{BL} & -62.5 \mathrm{~cm} \\ \mathrm{CBL} & +5.0 \mathrm{~cm}\end{array}$

TABIE XV

COORDINATION AND MOTOR ACCURACY: REPETITTVE

MONENENIS OF TYE KNEE

\begin{tabular}{|c|c|c|c|c|}
\hline GROUP & MEAN & RANGE & QUTCOME & \\
\hline$A L$ & +98 & $(-100$ to +448$)$ & $I=7 \quad W=3$ & \\
\hline CAL & -48 & $(-44$ to +228$)$ & $I=3 \quad W=1$ & \\
\hline AP & -138 & $(-100$ to +658$)$ & $I=4 \mathrm{~W}=6$ & \\
\hline CAP & -48 & $(-38$ to +268$)$ & $I=1 \quad W=1$ & $s=1$ \\
\hline $\mathrm{BP}$ & -58 & $(-32$ to +188$)$ & $I=3 \quad W=6$ & \\
\hline $\mathrm{CBP}$ & +258 & ( 0 to +498$)$ & $I=5$ & \\
\hline BL & -158 & $(-60$ to +238$)$ & $I=2 \quad W=6$ & $s=1$ \\
\hline CBI & +258 & $(-8$ to +598$)$ & $I=2 \quad W=I$ & $s=1$ \\
\hline
\end{tabular}

\begin{tabular}{|c|c|c|c|}
\hline STFGES & $\underline{n}$ & P (Safflower) & I (Lecithin) \\
\hline 0 & 8 & $+2.5 \mathrm{~cm}$ & $+3.7 \mathrm{~cm}$ \\
\hline I & 2 & $-15 \mathrm{~cm}$ & $-50 \mathrm{~cm}$ \\
\hline II & 11 & -30 & $-32.7 \mathrm{~cm}$ \\
\hline III & $1 *$ & -100 & -100 \\
\hline
\end{tabular}

- Patient lost ability to walk during the first six month period
TABLE XIV

COORDINATION AND MOIOR ACCURACY: REPETTTIVE MOVEMENTS OF THE FOOT

\begin{tabular}{|c|c|c|c|}
\hline GrOUP & MEAN & RANGE & artoge. \\
\hline ALl & -14.28 & $(-100$ to +130$)$ & $I=3 \quad W=6$ \\
\hline \multirow[t]{2}{*}{ ALr } & -27.28 & $(-100$ to +26$)$ & $I=3 \quad W=5$ \\
\hline & -20.78 & & \\
\hline API & -25.78 & $(-100$ to +46$)$ & $I=2 \quad W=8$ \\
\hline \multirow[t]{2}{*}{ APr } & -40.98 & $(-100$ to +20$)$ & $I=2 \quad W=8$ \\
\hline & -33.38 & & \\
\hline $\mathrm{BP} 1$ & +51.18 & $(-2$ to +275$)$ & $I=7 \quad W=1$ \\
\hline \multirow[t]{2}{*}{ RPX } & +8.98 & $(-17$ to +37$)$ & $I=6 \mathrm{~W}=3$ \\
\hline & +308 & & \\
\hline Bnl & +29.38 & $(-21$ to +187$)$ & $I=4 \quad W=3$ \\
\hline \multirow[t]{2}{*}{ Burr } & +31.78 & $(-50$ to +37$)$ & $I=7 \mathrm{~W}=\mathrm{i}$ \\
\hline & +20.58 & & \\
\hline STAGES & $\underline{n}$ & P (Safflower) & L (Lecithin) \\
\hline I & 2 & +11.68 & -2.18 \\
\hline II & 11 & -0.88 & -0.68 \\
\hline III & 8 & -5.48 & -3.38 \\
\hline IV & 1 & +0.68 & -7.88 \\
\hline
\end{tabular}

TABLE XVI

OOORDINATION AND MOTOR AOCURACY: REPETITIVE MOVDMENSS CF THE HIP

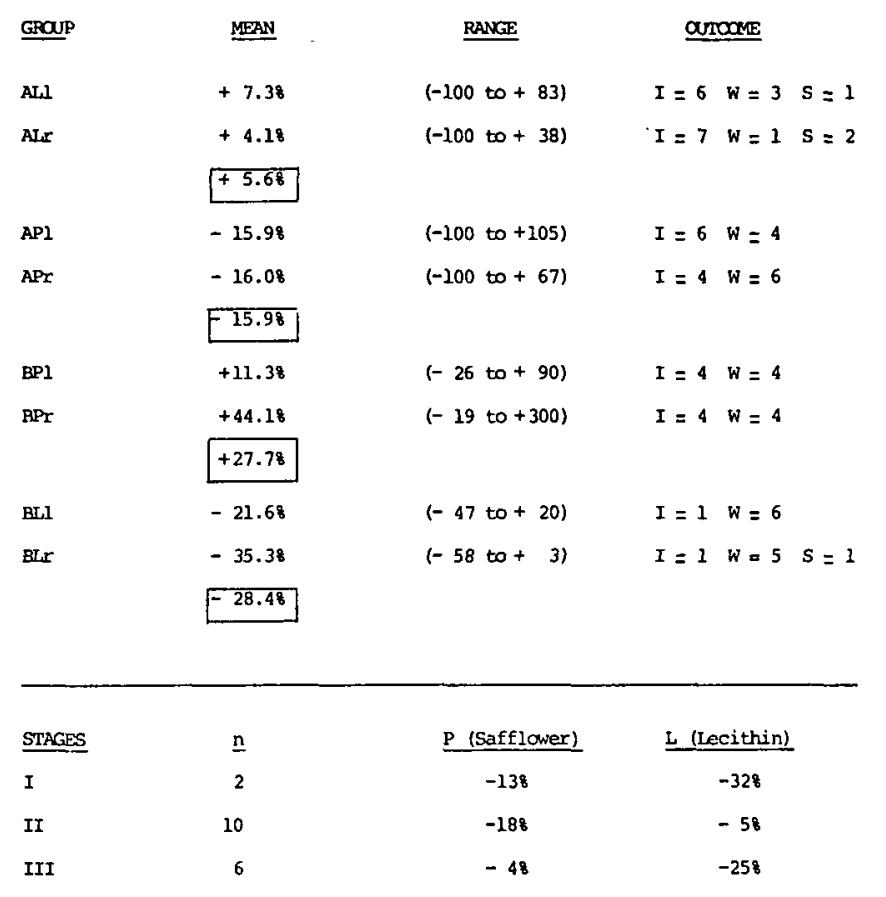


TARLE XNII

MFAN PERCENT CHANGE IN TIMED STABILITY (EQULIERTUM)

GROUP

TWO FEET TWO FEET

EYES CLOSED

EYES OPEN

EYES OPEN

AL,

AP

$-1.48$

$-7.08$

$+3.38$

$+0.58$

$+0.18$

BP

BL

$-1.68$

$-0.18$

$-1.38$

$+5.68$

$-0.68$

$-1.18$
TABIE XIX

COORDIVATION AND HOTOR ACCURACY: REPTTITTVE MOVDMENTS

OF THE FINGER (15 SEOONDS)

GROPP

MEAN

RANGE

OUTOONE

AL]

Alv

$-2.18$

$+0.58$

$(-33$ to +34$)$

$(-43$ to +62$)$

$-0.88$

AP1

APr

$+4.48$

$+9.58$

$(-34$ to +53$)$

$I=6 W=5$

$+6.98$

$-7.78$

$-21.38$

$-9.58$

$-2.38$

$-3.98$

$-3.18$
$(-27$ to +15$) \quad I=4 \quad W=6$

$(-27$ to +15$) \quad I=4 \quad W=6$

$(-40$ to +35$) \quad I=1 \mathrm{~W}=7 \quad \mathrm{~s}=2$

$(-35$ to +13$) \quad I=1 \quad W=9 \quad S=1$

$(-29$ to +30$) \quad I=5 \quad W=6$

$\begin{array}{lccccccc}\text { STAGES } & \underline{\underline{n}} & \underline{\mathrm{p}} & \underline{\mathrm{L}} & \underline{\mathrm{p}} & \underline{\mathrm{L}} & \underline{\underline{ }} & \underline{\mathrm{L}} \\ \text { I } & 2 & -5.48 & -5.88 & 08 & 08 & -0.38 & -2.28 \\ \text { II } & 11 & -0.78 & -0.58 & +3.68+1.88 & -0.28 & -0.38 \\ \text { III } & 1 & -2.08 & -2.08 & -21.08 & -21.08 & -1.08 & -1.08\end{array}$

TABLE XVIII

RESULTS OF THE SCSIT MOTOR ACOURACY TESTS ${ }^{\star}$ (\$ CHANGE)

$$
\text { ACCURACY SCORE }
$$

GROUP

MEAN QUTCOME

$I=6 \mathrm{~W}=4$

$$
I=6 \mathrm{~W}=4
$$$$
-7.5
$$

ADUUST'PD SCORE

AL $\quad-3.38$

$+17.08$

$I=5 \mathrm{~W}=6$

$-3.48$

$I=5 \mathrm{~W}=6$

BP $\quad-0.68$

$I=3 W=2$

$-7.48$

$I=5 W=5$

$+6.3 .48$

$$
\text { (n) }
$$

\begin{tabular}{lrccccc} 
STAGES & $\underline{\mathrm{n}}$ & $\underline{\mathrm{P}}$ & $\underline{\mathrm{L}}$ & $\underline{\mathrm{n}}$ & $\underline{\mathrm{P}}$ & $\underline{\mathrm{L}}$ \\
I & 2 & +55.98 & +99.58 & 2 & -1.68 & +20.88 \\
II & 11 & +19.78 & +31.68 & 10 & +3.38 & -6.78 \\
III & 7 & -24.88 & +20.48 & 8 & -16.98 & -12.38 \\
IV & 1 & +4.78 & +9.08 & 1 & +12.48 & -32.18 \\
\hline
\end{tabular}

* Western Psychological Services, 12031 wilshire Boulevard, Los Angeles, california.

\begin{tabular}{|c|c|c|c|}
\hline STRGES & $\underline{\mathbf{n}}$ & P (Safflower) & $\underline{L}$ (Iecithin) \\
\hline I & 2 & +11.68 & -2.18 \\
\hline II & 11 & -0.88 & -0.68 \\
\hline III & 8 & -5.48 & -3.38 \\
\hline IV & 1 & +0.68 & -7.88 \\
\hline
\end{tabular}

BLr

$$
-3.18
$$

\begin{tabular}{|c|c|c|c|c|}
\hline GROUP & MEAN & RANGE & OUTCOME & \\
\hline ALL & -0.48 & $(-17$ to +20$)$ & $I=5 \quad W=5$ & $s=1$ \\
\hline \multirow[t]{2}{*}{ Allr } & -3.68 & $(-19$ to +23$)$ & $I=3 \quad W=6$ & $s=2$ \\
\hline & -2.08 & & & \\
\hline API & +6.68 & $(-20$ to +50$)$ & $I=6 \quad W=5$ & \\
\hline \multirow[t]{2}{*}{ APr } & +3.48 & $(-11$ to +45$)$ & $I=5 \quad W=6$ & \\
\hline & +5.08 & & & \\
\hline BPI & +4.58 & $(-22$ to +36$)$ & $I=7 \quad W=3$ & $s=1$ \\
\hline \multirow[t]{2}{*}{$\mathrm{BPT}$} & -1.18 & $(-15$ to +17$)$ & $I=4 \quad W=6$ & $s=1$ \\
\hline & +1.78 & & & \\
\hline BLI & +4.58 & $(-7$ to +18$)$ & $I=6 \quad W=3$ & $s=2$ \\
\hline \multirow[t]{2}{*}{ Bi.r. } & -3.18 & $(-27$ to +27$)$ & $I=4 \quad W=5$ & $s=2$ \\
\hline & +1.28 & & & \\
\hline STAGES & $\underline{\mathrm{n}}$ & P (Safflower) & $\underline{L}$ (Lecithin & \\
\hline I & 2 & -0.48 & -3.58 & \\
\hline II & 11 & +5.48 & +0.58 & \\
\hline III & 8 & +3.68 & -14.58 & \\
\hline TV & 1 & -12.88 & +6.78 & \\
\hline
\end{tabular}

TABIE XX

UPPER EXTREMITIES: DISTAL COORDINATION (30 SEOONDS) 
TABLE XXI

UPPER EXTREMITIES: PROXIMAL COORDINATION (30 SEOONTS)

\begin{tabular}{|c|c|c|c|c|c|}
\hline Group & MEAN & RANGE & & TCOME & \\
\hline$A L l$ & -2.28 & $(-17$ to +18$)$ & $I=3$ & $w=5$ & $s=3$ \\
\hline \multirow[t]{2}{*}{ ALr } & +10.98 & $(-10$ to +85$)$ & $I=7$ & $w=3$ & $s=1$ \\
\hline & +6.58 & & & & \\
\hline AP1 & -2.58 & $(-17$ to +25$)$ & $I=4$ & $w=7$ & \\
\hline \multirow[t]{2}{*}{$\mathrm{APr}$} & +2.18 & $(-20$ to +33$)$ & $I=6$ & $w=5$ & \\
\hline & -0.28 & & & & \\
\hline $\mathrm{BPI}$ & -4.08 & $(-21$ to +37$)$ & $I=2$ & $W=8$ & $s=1$ \\
\hline \multirow[t]{2}{*}{$\mathrm{BP} x$} & -3.68 & $(-20$ to +18$)$ & $I=2$ & $W=5$ & $S=4$ \\
\hline & -3.88 & & & & \\
\hline BLI & -4.68 & $(-29$ to +22$)$ & $I=3$ & $\mathrm{w}=7$ & $s=1$ \\
\hline \multirow[t]{2}{*}{ BLr } & -2.28 & $(-13$ to +18$)$ & $I=3$ & $W=6$ & $S=2$ \\
\hline & -3.48 & & & & \\
\hline
\end{tabular}

\begin{tabular}{lccc}
\hline STAGES & $\mathbf{n}$ & $\frac{\mathrm{p} \text { (Safflower) }}{2 \text { L (Lecithin) }}$ \\
I & 2 & -1.28 & -6.18 \\
II & 11 & -1.28 & +0.88 \\
III & 8 & -1.38 & +1.38 \\
IV & 1 & -15.48 & +7.78 \\
\hline
\end{tabular}

TARIE XXII

UPPER EXTREMTTIES: METER-NOSE TEST (30 SHDONDS)

\begin{tabular}{|c|c|c|c|}
\hline Group & MEAN & RANGE & QuTCOME \\
\hline AL & +1.08 & $(-25$ to +278$)$ & $I=6 \quad W=4$ \\
\hline CAL & +9.58 & $(-15$ to +488$)$ & $I=2 W=2$ \\
\hline$A P$ & +2.58 & $(-22$ to +458$)$ & $I=6 \quad W=4$ \\
\hline CAP & +3.08 & $(-13$ to +338$)$ & $I=1 \quad W=2$ \\
\hline BP & -1.78 & $(-29$ to +258$)$ & $I=5 \quad W=6$ \\
\hline $\mathrm{CBP}$ & +2.08 & $(-15$ to +398$)$ & $I=2 \quad W=4$ \\
\hline BL. & -1.68 & $(-33$ to +338$)$ & $I=6 \quad W=5$ \\
\hline CRI. & +14.08 & ( o to +288$)$ & $I=4$ \\
\hline
\end{tabular}

\begin{tabular}{lrcc}
\hline SIPGES & $\underline{n}$ & $\frac{p \text { (Safflower) }}{+2.48}$ & $\frac{\text { L (Lecithin) }}{+11.88}$ \\
0 & 10 & -4.48 & -2.78 \\
I & 2 & +0.38 & +0.18 \\
II & II & +3.18 & -1.08 \\
III & 8 & -22.28 & +11.18 \\
IV & 1 & &
\end{tabular}

TARLE XOIIII

TRMED FUNCTIONAL TESTS

GROUP

SIX METER DRSH WITHOUT HANDRAI

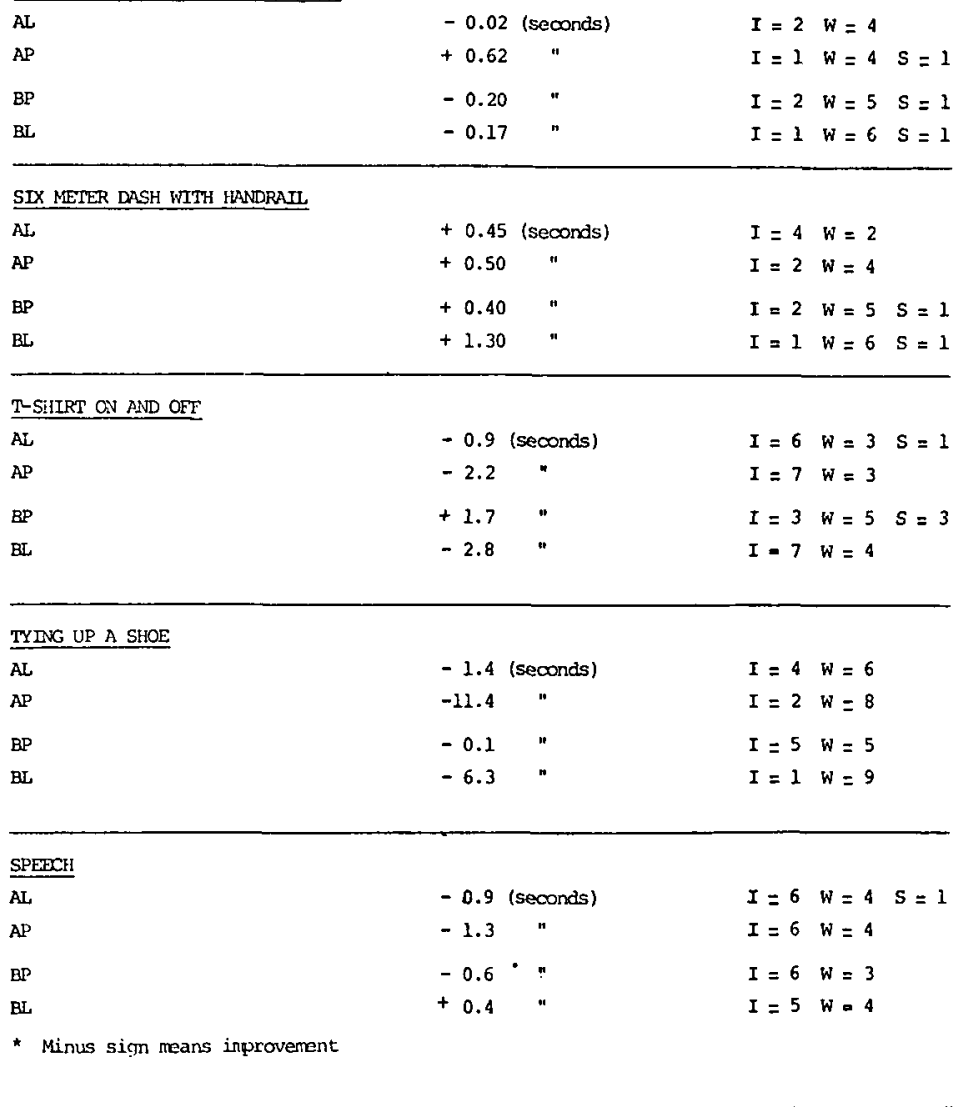


TABLE XXV

\section{UNTIMED FUNCTIONAL TESTS}

$\begin{array}{lcccc}\text { GROUP } & \text { NEAN } & \text { RANGE } & \text { OUTOCME } \\ \text { AL } & -9.28 & (-78 \text { to }+50) & I=3 \quad W=4 \quad S=4 \\ \text { AP } & -19.58 & (-78 \text { to }+29) & I=3 \quad W=5 \quad S=3 \\ \text { BP } & -10.48 & (-67 \text { to }+12) & I=2 \quad W=4 \quad S=5 \\ \text { BL } & -8.08 & (-38 \text { to }+6) & I=1 \quad W=3 \quad S=7\end{array}$

TARLE XXVI

RESULTS OF THE QUESTIONRAIRE: MEAN CHAITEE DN TOTAL SCORE

\begin{tabular}{llll} 
GROUP & MEAN & RANGE & \multicolumn{2}{c}{ OUTCOME } \\
AL & +3.08 & $(-36$ to +358$)$ & $I=6 \quad \mathrm{~W}=3 \quad \mathrm{~S}=2$ \\
$\mathrm{AP}$ & +4.38 & $(-43$ to +368$)$ & $\mathrm{I}=7 \mathrm{~W}=4$ \\
$\mathrm{BP}$ & -5.98 & $(-39$ to +508$)$ & $\mathrm{I}=3 \mathrm{~W}=7 \mathrm{~S}=1$ \\
$\mathrm{BL}$ & -8.98 & $(-50$ to +628$)$ & $\mathrm{I}=2 \mathrm{~W}=7 \mathrm{~S}=2$
\end{tabular}

$\begin{array}{lrrc}\text { STAGES } & \underline{n} & \underline{\text { P (Safflower) }} & \text { L. (Lecithin) } \\ \text { I } & 2 & -58 & -128 \\ \text { II } & 11 & -18 & -28 \\ \text { III } & 8 & +38 & -18 \\ \text { IV } & 1 & -128 & -128\end{array}$

TABLE XXVII

RESULTS OF TTE QUESTIONNAIRE ACOORDING TO STAGES

AND SPECIFIC CRITERIA

STAGES TRANSFER FEEDING HYGIENE CIOTHNG SPEBCH WRTTING TOTAL $+1-+1-+1-+1-+1-2+1-$

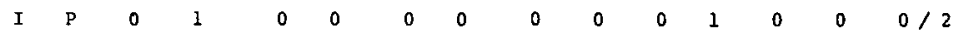

$\begin{array}{llllllllllllll}\mathrm{L} & 0 & 1 & 0 & 0 & 0 & 1 & 0 & 1 & 0 & 1 & 0 & 1 & 0 / 5\end{array}$

\begin{tabular}{lllllllllllllll}
\hline II & $P$ & 3 & 3 & 0 & 1 & 1 & 2 & 3 & 2 & 3 & 3 & 2 & 1 & $12 / 12$
\end{tabular}

$\begin{array}{llllllllllllll}\text { L } & 1 & 5 & 1 & 1 & 2 & 3 & 3 & 2 & 4 & 2 & 4 & 1 & 15 / 14\end{array}$

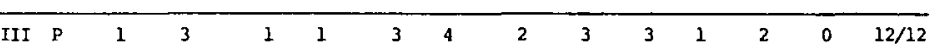

$\begin{array}{llllllllllllll}\mathbf{L} & 2 & 4 & 1 & 0 & 3 & 3 & 3 & 3 & 1 & 1 & 2 & 0 & 11 / 11\end{array}$

\begin{tabular}{|c|c|c|c|c|c|c|c|c|c|c|c|c|c|c|}
\hline \multirow{2}{*}{$\mathrm{IN}$} & $\mathrm{P}$ & 0 & 1 & 0 & 0 & 0 & 0 & 0 & 0 & 0 & 0 & 0 & 0 & $0 / 1$ \\
\hline & L & 0 & 1 & 0 & 0 & 0 & 0 & 0 & 0 & 0 & 0 & 0 & 0 & $0 / 1$ \\
\hline
\end{tabular}

TOTAL

$9 / 13$

$11 / 11$

$11 / 9$

$10 / 350 / 58$

TABLE XXVIII

SUMAARY OF CIMNGES IN ALL CINIICAL PARANETERS DN 22 PATIENIS WITH FRIEDREICI!'S ATAXLA NFTER A SLX-HOMTY TRIAL OF LECITHEN

\section{OR SAFFIOWFR OII}

TESTS

JOLTT MOBILITY

AEAN GAJN (4) OR LOSS (-) FROM
PRE-TRLiL SOORES
$\begin{aligned} & \text { P (SAFFLONER) } \\ & \text { L (LNCITHIN) }\end{aligned}$

MSCLE STRENGSH

Scmi-quantitative

Dynamoneter

$-0.68$

$-3.58$

LONZR ENTPEMITIIS

Gait

Neter tersts

Stability

$-0.18$

$-3.18$

$+47.08$

$+56.08$

UPPER EXTFLMITIES

Motor accuracy

Mater tests

$-29.3 \mathrm{~cm}$

-36.2 वा

$-1.68$

$-4.98$

$-0.18$

$-0.88$

FUNCTYONAL TESTS

Tjmed

Untimed

$+7.48$

$+11.38$

$+0.18$

$-0.38$

$-1.28$

$-1.08$

$-19.28$

$-8.68$

QUESTIONALIRE

$-2.98$ 
strates that only two clinical features, i.e. muscle strength and motor accuracy in the upper extremities have kept positive values in both trials of lecithin and safflower oil. There was however no evidence of significant difference between lecithin and safflower oil in the mean gain or loss in any specific clinical feature.

\section{DISCUSSION}

Contrary to previous reports of early and significant improvement in muscle strength and motor coordination with lecithin in patients with Friedreich's and other forms of spino-cerebellar ataxia (Barbeau, 1979: Livingstone et al, 1981) this study failed to demonstrate any beneficial effect, in any specific clinical parameter, which could be attributed to lecithin itself. Our results have showed that some patients improved with lecithin. These patients were mostly younger individuals from functional stage $I$ and their level of improvement never passed control values. The only patient who surpassed normal control values in some specific muscle function tests while on lecithin, was our single stage IV patient. It is difficult to conclude in favour of lecithin under these circumstances.

The usefulness of a control group in such a trial, needs to be emphasized. Although our control group was small in comparison with the number of patients followed, we have gained some precious information from their contribution. First, contrary to patients, all mean scores for tests administered to controls resulted in positive values. Second, some individual controls lost points in specific tests, after either lecithin or safflower oil, in the same way some patients did. Third, we observed no difference in mean improvement scores for controls, whether on lecithin or safflower oil. Fourth, controls as well as patients learned to improve their performance, as demonstrated by the increment of test scores at the end of the second period of the trial in both groups.

Without the control group, it would have seemed logical to conclude that lecithin led to a $56 \%$ improvement in muscle strength and a $11 \%$ gain in coordination in the upper extremities in patients with Friedreich's Ataxia (table XXVIII). It is however more difficult to explain why patients on safflower oil, which does not contain phosphatidyl choline, had better scores in 7 out of 11 clinical parameters tested, and demonstrated a mean of $8 \%$ less deterioration in all clinical features other than muscle strength and coordination (table XXVIII).

Safflower oil contains $30 \%$ more linoleic acid per weight than lecithin (see previous article in this issue). The implication of linoleic acid in the composition of mitochondrial membranes and as a precursor of lipoic acid has been thoroughly reviewed by Barbeau (1980). It has not yet been established that previous observations of clinical improvement in Friedreich's Ataxia relates to the choline moiety rather than the linoleic acid-rich composition of lecithin. In view of the associated defects in the pyruvate and alphaketoglutarate dehydrogenase enzyme systems, which rely on lipoic acid as a cofactor, it would seem possible that the previously reported improvement in symptomatology in Friedreich's Ataxia with lecithin and our observed comparable effect with safflower oil proceed from the same basic principle, i.e. increased supply of linoleic acid to mitochondrial membranes and energy metabolism.

In conclusion, this study has fulfilled its two main objectives. With regards to (1) methods of clinical assessment, we believe that metered tests and timed functional tests provided the most reliable results. As for (2) concordance between objective tests results and patient's subjective feeling of changes in their clinical status, we noticed good correlation for most functions with the exception of transfer and writing. Finally we acknowledge the need for another clinical study in which the effect of pure linoleic acid alone would be assessed in patients with Friedreich's Ataxia and normal controls.

\section{ACKNOWLEDGEMENTS}

This clinical trial was possible owing to grants from l'Association Canadienne de l'Ataxie de Friedreich, the Muscular Dystrophy Association of Canada and the Quebec Network of Medical Genetics. We particularly want to thank Luc St-Jean, Solange Lebrun St-Jacques and the parents of our patients and controls.

\section{REFERENCES}

BARBEAU, A. (1979). Lecithin in movement disorders. In: Barbeau, A., Growdon, J.H., Wurtman, R.J. eds. Nutrition and the brain. Vol. 5, New York: Raven Press, 263-271.

BARBEAU, A. (1980). Friedreich's Ataxia 1980. An overview. Can. J. Neurol. Sci., 7: 455-468.

LIVINGSTONE, I.R., MASTAGLIA, F.L., PENNINGTON, R.J.T. and SKILBECK, C. (1981). Choline chloride in the treatment of cerebellar and spinocerebellar ataxia. $\mathbf{J}$. Neurol. Sci., 50: 161-174.

PENTLAND, B., MARTYN, C.N. STEER, C.R. and CHRISTIE, J.E. (1981). Lecithin in Friedreich's ataxia. Br. Med. J. 282: 1197-1198. 